\title{
Eficacia y seguridad de una regla de decisión para orientar el tratamiento en niños con neumonía vacunados contra neumococo. Un ensayo clínico controlado
}

\author{
Efficacy and safety of a decision rule for using antibiotics \\ in children with pneumonia and vaccinated against \\ pneumococcus. A randomized controlled trial
}

\author{
Dr. Fernando Ferrero ${ }^{a}$, Dr. Fernando Adrián Torres ${ }^{b}$, Dra. Paula Domínguez $z^{c} y$ \\ Dra. María Fabiana Ossorio ${ }^{b}$
}

\section{RESUMEN}

Introducción. A pesar de que la mayoría de las neumonías en menores de 5 años son virales, en la práctica diaria, frecuentemente, son tratadas con antibióticos. Una regla clínica de decisión (BPS: Bacterial Pneumonia Score) demostró ser eficaz para identificar qué niños con neumonía requerían antibióticos, pero su desempeñono ha sido evaluado en la población vacunada contra neumococo.

El objetivo fue evaluar si el empleo del BPS permitía un menor uso de antibióticos comparado con el manejo habitual en niños con neumonía adquirida en la comunidad, que recibieron vacunación antineumocóccica.

Material y método. Ensayo clínico controlado, aleatorizado, de grupos paralelos, con enmascaramiento parcial, que compara dos métodos de manejo de niños de 3-60 meses de edad asistidos ambulatoriamente por neumonía, que hubieran recibido vacuna antineumocóccica conjugada. El Grupo BPS recibió antibióticos con $\mathrm{BPS} \geq 4$ puntos; el grupo control recibió antibióticos según criterio del médico tratante. El tamaño muestral calculado contempló, al menos, 30 pacientes por grupo. Se comparó la proporción de uso de antibióticos y la evolución clínica en ambos grupos.

Resultados. Se incluyeron 65 pacientes (33 en el grupo BPS y 32 en el grupo control), con edad promedio de 17,5 meses. El empleo de antibióticos fue significativamente mayor en el grupo control que en el grupo BPS (21/32 vs. 9/33; OR 5,09; IC 95\%: 1,57-16,85; p = 0,001). Se observó una mala evolución en 7 pacientes ( 3 del grupo BPS y 4 del grupo control).

Conclusión. El empleo de BPS permitió un menor uso de antibióticos para el manejo inicial de pacientes con neumonía vacunados contra neumococo, sin aumentar el riesgo de mala evolución.

Palabras clave: neumonía, infecciones del sistema respiratorio, técnicas de apoyo para la decisión.

http:/ /dx.doi.org/10.5546/aap.2015.397

\section{INTRODUCCIÓN}

La neumonía continúa siendo una importante causa de morbimortalidad infantil, con una incidencia anual de 36-40 episodios / 1000 menores de 5 años. ${ }^{1}$

A pesar de que, en este grupo etario, la mayoría es de etiología viral, ${ }^{2}$ al no poder contar con un diagnóstico etiológico durante la consulta, se corre riesgo de sobrediagnosticar infección bacteriana y, consecuentemente, indicar un tratamiento antibiótico innecesario. Esto ocasiona un incremento de la resistencia bacteriana, de los costos y del riesgo de efectos adversos. ${ }^{3}$

Se puede recurrir a indicadores indirectos de etiología, que incluyen elementos epidemiológicos (época del año, edad), clínicos (temperatura, signos respiratorios), de laboratorio (leucocitos, velocidad de sedimentación globular) e imágenes (radiografía de tórax), pero ninguno en forma aislada tiene capacidad efectiva para predecir la etiología.

Los llamados modelos de predicción clínica, que combinan varios elementos, han sido empleados exitosamente con fines diagnósticos o pronósticos en diversas situaciones clínicas. ${ }^{4}$ En 2006, Moreno y col. diseñaron un modelo predictivo (Bacterial Pneumonia Score o BPS), que permitía identificar con precisión a los pacientes con neumonía que no se beneficiarían con antibióticos. ${ }^{5}$ 
Esta herramienta fue adecuadamente validada en forma prospectiva e, incluso, valorada en pacientes ambulatorios. ${ }^{6}$ Más aún, su impacto en el uso de antibióticos fue valorado a través de un ensayo clínico controlado, ${ }^{7}$ y se cumplió así con el mejor estándar para el desarrollo de este tipo de herramientas para el manejo clínico. ${ }^{8,9}$

Sin embargo, todos los estudios que incluyeron el desarrollo, validación y valoración del impacto del BPS se desarrollaron antes de 2012. En 2012, se incorporó al calendario oficial de inmunizaciones de Argentina la vacuna antineumocóccica conjugada (Prevenar 13 $3^{\mathrm{MR}}$ ) para menores de 2 años. Existe evidencia de que su introducción masiva puede modificar considerablemente el patrón epidemiológico de la enfermedad. ${ }^{10,11}$ Es razonable pensar que la vacunación podría tener alguna influencia en el desempeño que esta regla de predicción ha mostrado.

El objetivo del presente trabajo fue evaluar si el empleo de la regla clínica de decisión BPS para el manejo inicial de pacientes con neumonía permitía un menor uso de antibióticos que el manejo habitual de esta patología, sin incrementar la proporción de fallas del tratamiento, en una población con cobertura de vacuna antineumocóccica conjugada.

\section{POBLACIÓN Y MÉTODOS}

Estudio clínico controlado, aleatorizado, de grupos paralelos, con enmascaramiento parcial, que compara dos métodos de manejo (BPS y manejo habitual) de pacientes con neumonía.

Se incluyeron pacientes de 3-60 meses de edad asistidos en consultorios externos del Hospital General de Niños Pedro de Elizalde (HGNPE) con diagnóstico clínico de neumonía (fiebre, tos, taquipnea y auscultación compatible), ${ }^{12}$ con 24-48 horas de evolución, inmunización antineumocóccica conjugada completa para la edad y consentimiento informado firmado. Se excluyeron aquellos con sibilancias, neumonía grave y muy grave, ${ }^{1}$ vacunación incompleta, enfermedad cardíaca o pulmonar crónica, derrame pleural, absceso pulmonar, necesidad de internación, insuficiencia hepática o renal, síndrome de Down, tratamiento antibiótico o internación en las dos semanas previas.

El estudio se extendió durante un año (del $1 / 4 / 2013$ al 31/3/2014).

Todos los pacientes que, en el consultorio de orientación del HGNPE, fueron diagnosticados con neumonía se remitieron a un investigador para su evaluación. Se invitó a participar a aquellos que cumplieron con todos los criterios de inclusión y ninguno de exclusión. Luego, se asignó a cada paciente el tipo de manejo (BPS o manejo habitual). La aleatorización estuvo a cargo del investigador, mediante sobre cerrado.

Los pacientes del grupo control fueron remitidos al sistema de turnos que les asignó un médico tratante, quien decidió el manejo, los exámenes complementarios y el eventual uso de antibióticos, de acuerdo con la guía de práctica clínica de la Institución, que considera el tratamiento empírico inicial con antibióticos (amoxicilina: $80-100 \mathrm{mg} / \mathrm{kg} /$ día) para todo paciente menor de 5 años con neumonía de manejo ambulatorio en que se considerara una posible etiología bacteriana. ${ }^{13}$

Los pacientes asignados al grupo estudio (BPS) continuaron con el investigador y se les realizó un examen clínico, medición de temperatura axilar, hemograma y radiografía de tórax, y se calculó con ellos el valor de BPS. Esta regla presenta un rango de -3 a 15 puntos $^{5}$ (Figura 1). Se indicó antibiótico (amoxicilina: $80-100 \mathrm{mg} / \mathrm{kg} /$ día) ${ }^{14}$ a aquellos con BPS $\geq 4$ puntos. En pacientes con BPS $<4$, se mantuvo una conducta expectante.

En ambos grupos, los pacientes fueron citados luego para control por otro investigador, ciego al grupo de tratamiento, quien valoró la evolución clínica dentro de las primeras $24 \mathrm{~h}$, a las $48 \pm 12$ h, $5 \pm 1$ día, $7 \pm 1$ día y $10 \pm 1$ día, y verificó cada vez las medidas de resultado principal (uso de antibióticos) y secundaria (evolución clínica). En todo paciente que no cumpliera criterios de buena evolución clínica, se indicó antibiótico de primera línea si no lo estuviera recibiendo, de segunda línea si ya lo estaba recibiendo, o bien internación, de considerarse necesario.

La variable de resultado primaria fue proporción de utilización de antibióticos en ambos grupos. La variable de resultado secundaria fue evolución clínica, definida como buena cuando cumpliera con todos los siguientes criterios: disminución de la fiebre antes de $48 \mathrm{~h}$, frecuencia respiratoria normal o disminución mayor de 5 respiraciones/minuto antes de $48 \mathrm{~h}$, ausencia de signos de neumonía grave, no haber necesitado internación ni incorporación de antibióticos o rotación de estos si los había requerido inicialmente. La evaluación final se efectuó al $7^{\circ}$ día. Se consideró la variable como categórica (evolución clínica buena o mala).

Para evaluar si ambos grupos eran comparables, se utilizaron como variables de control edad, sexo y temperatura axilar. 
Tamaño muestral: Esperando disminuir $\geq 40 \%$ la tasa de utilización de antibióticos entre el grupo BPS y el grupo control ( $86 \%$ vs. $46 \%){ }^{7}$ se estimó una muestra de 26 pacientes por grupo, con una potencia de $80 \%$ y error alfa a dos colas de 0,05 . Se contempló reclutar 30 sujetos por grupo ante eventuales pérdidas para el seguimiento y extender el estudio, al menos, un año para evitar sesgos epidemiológicos.

Análisis estadístico: Se describió la distribución de los valores mediante media y desvío estándar para variables numéricas y porcentajes con IC 95\% para variables categóricas. Para comparar ambos grupos, se utilizó la prueba de t para muestras independientes y la prueba de Chi cuadrado, según correspondiere. Se calculó la proporción de utilización de antibióticos y la mala evolución clínica en cada grupo y se compararon con la prueba de Chi cuadrado. Se calcularon OR con IC $95 \%$. En todos los casos, se tomó un nivel de significación de $\mathrm{p}<0,05$. Se utilizó SPSS 11.0, Chicago, EE. UU., 2001.

Consideraciones éticas: El estudio se guió por las normas de buenas prácticas clínicas, la Declaración de Helsinki y la normativa comunal y nacional vigente. Se contó con la aprobación de los comités institucionales. Se solicitó y obtuvo consentimiento informado de todos los participantes. Se contó con un Comité de Monitoreo de Seguridad, que evaluó la aparición de efectos adversos graves y, periódicamente, la proporción de fallas al tratamiento. El estudio fue registrado en Clinicaltrials.gov (NCT01875731).

\section{RESULTADOS}

Se incluyeron 65 pacientes (grupo BPS: 33; grupo control: 32) (Figura 2). Treinta y dos fueron mujeres; la edad promedio fue 17,5 $\pm 10,5$ meses (rango: 3,3-49 meses). La temperatura axilar promedio fue $38,5 \pm 0,4{ }^{\circ} \mathrm{C}$ (rango: $38-39,2^{\circ} \mathrm{C}$ ). Más de la mitad de los pacientes habían recibido 3 dosis de vacuna (la tercera correspondió al refuerzo) (Tabla 1$)$.

No se observaron diferencias entre los pacientes del grupo BPS y del grupo control en lo relacionado con la edad $(17,2 \pm 10,2$ meses vs. $17,5 \pm 11$ meses; $p=0,83)$, temperatura axilar $\left(38,5 \pm 0,4{ }^{\circ} \mathrm{C}\right.$ vs. $\left.38,6 \pm 0,4{ }^{\circ} \mathrm{C} ; \mathrm{p}=0,23\right) \mathrm{ni}$ distribución por género (varones: $19 / 33$ vs. 14/32; OR 1,06; IC 95\%: 0,35-3,06; $p=0,88$ ) (Tabla 1).

El análisis de BPS mostró un puntaje mínimo de -2 y un máximo de $9 ; 24 / 33$ pacientes tuvieron un puntaje menor de 4 puntos.

El 46\% (30/65) de los pacientes recibieron tratamiento antibiótico. La utilización de antibióticos en el manejo inicial de neumonía

FIgURA 1. BPS (Bacterial Pneumonia Score). Regla de decisión para el uso de antibióticos en niños menores de 5 años con neumonía adquirida en la comunidad

\begin{tabular}{|c|c|c|c|}
\hline \multicolumn{3}{|l|}{ Predictor } & Puntos \\
\hline \multicolumn{3}{|c|}{ Temperatura axilar $\geq 39^{\circ} \mathrm{C}$} & 3 \\
\hline \multicolumn{3}{|c|}{ Edad $\geq 9$ meses } & 2 \\
\hline \multicolumn{3}{|c|}{ Recuento absoluto de neutrófilos $\geq 8000 / \mathrm{mm}^{3}$} & 2 \\
\hline \multicolumn{3}{|c|}{ Neutrófilos en banda $\geq 5 \%$} & 1 \\
\hline \multirow[t]{13}{*}{ Radiografía } & Infiltrado & Bien definido, lobular, segmentario & 2 \\
\hline & & Pobremente definido, parches & 1 \\
\hline & & Intersticial, peribronquial & -1 \\
\hline & Localización & Un lóbulo & 1 \\
\hline & & $\begin{array}{l}\text { Múltiples lóbulos en uno o ambos pulmones, } \\
\text { pero bien definidos }\end{array}$ & 1 \\
\hline & & Múltiples sitios, peribronquial y mal definidos & -1 \\
\hline & Derrame pleural & Mínimo & 1 \\
\hline & & Claro & 2 \\
\hline & Absceso, bullas o pneumatocele & Dudoso & 1 \\
\hline & & Claro & 2 \\
\hline & Atelectasia & Subsegmentaria & -1 \\
\hline & & Lobar con compromiso de lóbulos medio o superior derecho & -1 \\
\hline & & Lobar con compromiso de otros lóbulos & 0 \\
\hline \multicolumn{3}{|l|}{ Puntaje total } & De -3 a 15 \\
\hline
\end{tabular}

BPS (Bacterial Pneumonia Score) $\geq 4$ puntos = indicación de antibióticos. 
se observó en 9/33 pacientes del grupo BPS y en 21/32 del grupo control (OR: 5,09; IC 95\%: $1,57-16,85 ; p=0,001$ ).

La evolución fue desfavorable en $7 / 65$ pacientes $(10,7 \%)$ (otitis media aguda: 3 ; gastroenteritis aguda: 4). Los pacientes con evolución desfavorable correspondieron 3 al grupo BPS y 4 al grupo control (OR 0,7; IC 95\%: $0,1-4,19 ; \mathrm{p}=0,71)$. Respecto de los pacientes con evolución desfavorable, 4 no habían recibido antibióticos (2 de cada grupo).

\section{DISCUSIÓN}

Los pacientes con neumonía, vacunados contra neumococo, cuyo tratamiento fue decidido sobre la base del BPS, recibieron antibiótico mucho menos frecuentemente que aquellos tratados según el criterio del médico tratante. Esta manifiesta disminución en el uso de antibióticos se verificó sin incrementar la tasa de fracaso del tratamiento.

A pesar de que existe sobrada evidencia de que una considerable proporción de las neumonías en la infancia son ocasionadas por infecciones virales, ${ }^{15}$ las principales guías de práctica clínica incluyen el uso empírico y sistemático de antibióticos. Esto era particularmente evidente en las guías de la Organización Mundial de la Salud (OMS), ${ }^{12}$ en las que taxativamente se especificaba que todo niño con neumonía debía recibir antibióticos. Luego, tanto la Sociedad Argentina de Pediatría $(\mathrm{SAP}){ }^{14}$ como la British Thoracic Society (BTS), ${ }^{1}$ la Infectious Disease Society of America (IDSA) ${ }^{2}$ y la American Academy of Pediatrics ${ }^{16}$ incorporaron, en sus respectivas normas, consideraciones sobre la elevada prevalencia de etiología viral en las neumonías en la infancia, particularmente en los dos primeros años de vida. Sin embargo, todos los documentos coinciden en la necesidad de emplear antibióticos ante la imposibilidad de efectuar un diagnóstico etiológico.

Esta dificultad para identificar adecuadamente la etiología de las neumonías en niños ha dado lugar a un importante uso inapropiado de antibióticos, que incrementa la resistencia bacteriana, los costos de salud y la prevalencia de efectos adversos.

Existe evidencia de que una proporción

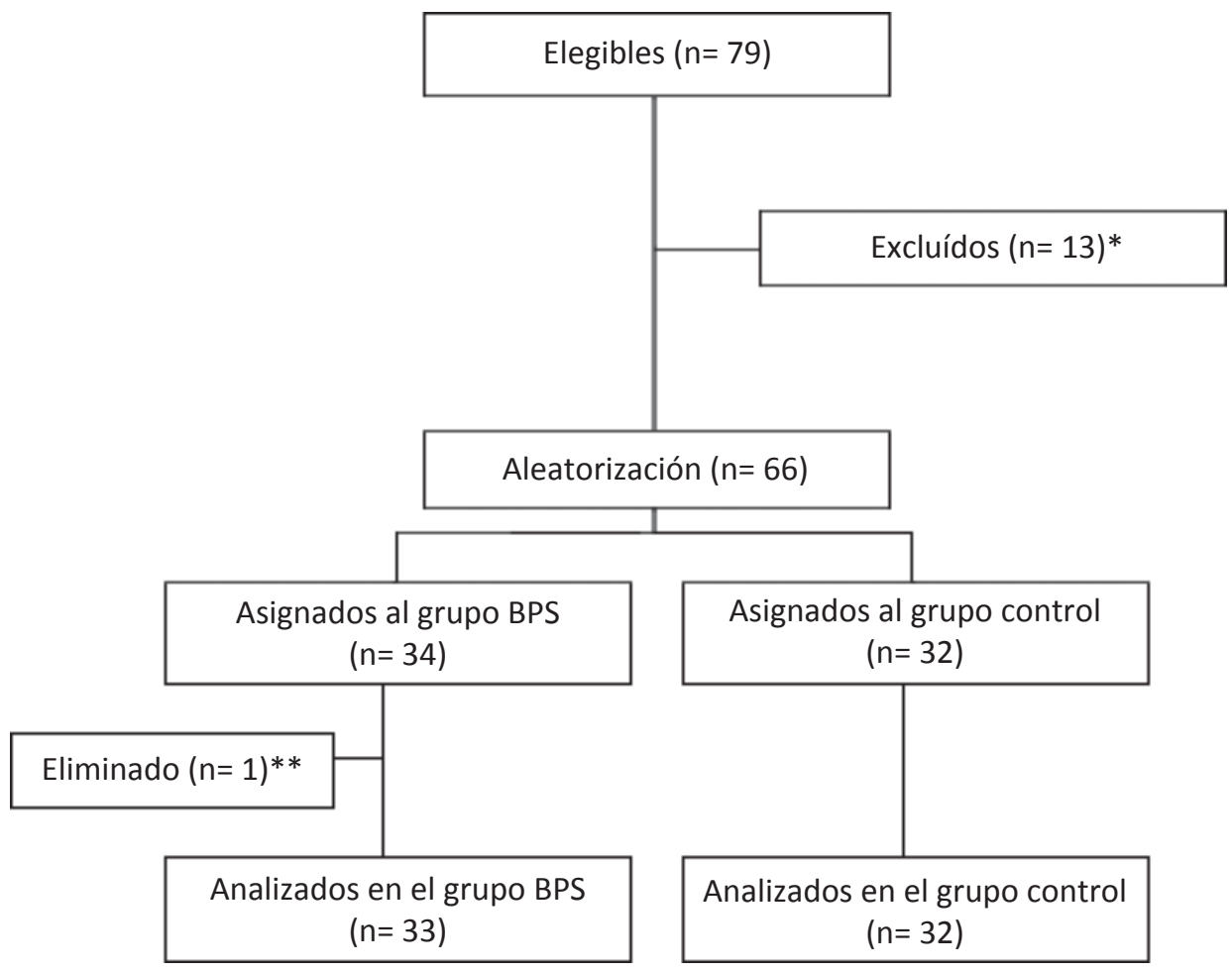

* Edad (2), exantema (1), falta carnet (5), prematuro (1), vacunas incompletas (4).

** No se pudo efectuar determinación de laboratorio. 
significativa de los niños que sufren infección respiratoria aguda reciben antibióticos, a pesar de tratarse de cuadros virales en muchos casos. En Argentina, $90 \%$ de las neumonías ${ }^{17}$ y $48 \%$ de las bronquiolitis fueron tratadas con antibióticos. ${ }^{18}$ No es muy distinto de lo que ocurre en Estados Unidos de América, donde la mayoría de las neumonías adquiridas en la comunidad en niños son tratadas con antibióticos y el $80 \%$ reciben inadecuadamente antibióticos de amplio espectro. ${ }^{19}$

Existe evidencia de que el uso de antibióticos en infecciones respiratorias incrementa 4 veces la portación de microorganismos resistentes a esos antibióticos y crean las condiciones para el uso de antibióticos de segunda línea. ${ }^{20}$ Por lo contrario, se ha comprobado que disminuir el empleo innecesario de antibióticos puede contribuir a disminuir la resistencia bacteriana. ${ }^{21}$

La magnitud del problema, lejos de estabilizarse, muestra un peligroso y continuo incremento. El número de hospitalizaciones por infecciones en las que se verificó resistencia antibiótica se incrementó en $359 \%$ en el decenio que va de 1997 a 2006, y muestra un mayor aumento en los menores de 18 años. ${ }^{22}$ Considerando los valores del National Committee on Clinical Laboratory Standards (NCCLS) anteriores a 2008, la resistencia a la penicilina del Streptococcus pneumoniae se incrementó en la mayoría de los países de Latinoamérica y, en Argentina, se duplicó (de 20,6\% a 44,4\%) entre 1994 y $1998 .{ }^{23}$ Afortunadamente, este aumento de la resistencia "in vitro" no incrementó el riesgo de fracaso al tratamiento con penicilina en niños hospitalizados por neumonía. ${ }^{24}$
Basar el diagnóstico de neumonía solo en elementos clínicos (tos y taquipnea) ${ }^{12}$ implica que muchos niños que presentan radiografía normal sean diagnosticados como con neumonía. ${ }^{25}$ La radiografía de tórax constituye un método de diagnóstico complementario fácilmente accesible y de bajo costo, que puede colaborar en incrementar la especificidad del diagnóstico.

Si bien la capacidad de la radiografía de tórax para predecir la etiología de la neumonía ha sido cuestionada, ${ }^{26}$ se incrementa con el empleo de formas de evaluación estandarizadas. En este sentido, Swingler ${ }^{27}$ reconoce que la forma de evaluar la radiografía de tórax establecida por Khamapirad ${ }^{28}$ es la única con capacidad para distinguir entre etiología viral y bacteriana. Esta forma de evaluar la radiografía de tórax ha demostrado una adecuada capacidad diagnóstica para identificar la neumonía bacteriana ${ }^{29}$ y es la que emplea el BPS.

Puede considerarse el grado de acuerdo entre observadores como una evaluación indirecta de la capacidad diagnóstica de la radiografía de tórax..$^{30}$ La interpretación mediante una forma simple y estandarizada permite unificar criterios $\mathrm{y}$, en consecuencia, obtener un buen grado de acuerdo entre observadores. ${ }^{31}$ Además, el método de Khamapirad ha demostrado una excelente especificidad, ${ }^{32}$ probablemente explicada por los puntos negativos que en algunos casos otorga (ejemplo: infiltrado intersticial, atelectasias) (Figura 1).

Por último, a diferencia de aquellos métodos que se basan exclusivamente en la radiografía de tórax para inferir la etiología de la neumonía, ${ }^{33}$ el BPS incorpora elementos clínicos y de laboratorio

TABLA 1. Comparación entre el grupo estudio y el grupo control

\begin{tabular}{lccc}
\hline & $\begin{array}{c}\text { Grupo estudio } \\
(\mathbf{n}=33)\end{array}$ & $\begin{array}{c}\text { Grupo control } \\
\text { (n = 32) }\end{array}$ & Significación \\
\hline Género (masculino) & $19 / 33$ & $14 / 32$ & $0,88^{*}$ \\
Edad (meses) & $17,2 \pm 10,2$ & $17,5 \pm 11$ & $0,83^{* *}$ \\
Temperatura $\left({ }^{\circ} \mathrm{C}\right)$ & $38,5 \pm 0,4$ & $38,6 \pm 0,4$ & $0,23^{* *}$ \\
Número de dosis de vacuna antineumocóccica & & - & - \\
$\quad$ - 1 dosis & 2 & 10 & - \\
$\quad$ - dosis & 8 & 22 & - \\
Uso desis ${ }^{* * *}$ & 23 & $21 / 32$ & $0,001^{*}$ \\
Evolución desfavorable & $9 / 33$ & $4 / 32$ & $0,71^{*}$ \\
\hline
\end{tabular}

* Prueba de Chi cuadrado.

** Prueba de t para muestras independientes.

*** Última dosis corresponde a dosis de refuerzo. 
que incrementan la especificidad. ${ }^{34}$

A pesar de que existen varias reglas clínicas de predicción orientadas a la salud infantil, pocas de ellas son usadas en la práctica diaria. Es posible que su limitado empleo esté relacionado con la errónea expectativa de que las reglas clínicas de predicción deben tener $100 \%$ de sensibilidad (cuando muy pocas tienen más de $90 \%$ ). Es importante recordar que una regla clínica de predicción adecuadamente construida y validada, aunque diste de la perfección, siempre será más sensible que el criterio clínico por sí solo. ${ }^{35}$ Más aún, si, como en el caso del BPS, se ha procedido a transformar la herramienta de predicción en una de decisión por medio de un estudio clínico controlado.

Nuestro estudio presenta fortalezas que deben ser tenidas en cuenta. Por un lado, su desarrollo durante un año completo ha permitido disminuir el sesgo que la circulación ocasional de varios microorganismos podría imprimir si se hubieran considerado períodos menores. Además, se eligió el mejor diseño (aleatorizado y enmascarado al investigador) para comprobar el desempeño de una regla de decisión clínica ${ }^{8,9}$ y una potente medida de resultado que no dejara lugar a dudas a la hora de valorar la eficacia del empleo de la herramienta en estudio. Finalmente, se logró un excelente seguimiento de los pacientes (sin pérdidas), que garantiza la seguridad del empleo del BPS, en el escenario elegido.

Por otro lado, el estudio presenta potenciales debilidades, que deben ser consideradas. No se efectuó una pesquisa microbiológica en ningún paciente; sin embargo, no está contemplada en ninguna guía para el manejo del paciente con neumonía asistido en forma ambulatoria. ${ }^{15,16}$ De igual modo, el estudio se desarrolló en un hospital especializado, pero todos los pacientes habían concurrido por demanda espontánea y todos eran pasibles de tratamiento ambulatorio inicialmente.

Por último, el empleo sistemático del BPS podría colaborar en contener el uso inadecuado de antibióticos y sus consecuencias (resistencia, costos, efectos adversos), inclusive en niños vacunados contra neumococo.

\section{CONCLUSIÓN}

Los pacientes con neumonía vacunados contra neumococo, cuyo tratamiento fue decidido sobre la base del BPS, recibieron antibióticos en la mitad de las oportunidades que aquellos tratados según el criterio del médico. Esta manifiesta disminución en el uso de antibióticos se verificó sin incrementar la tasa de fracaso del tratamiento.

\section{REFERENCIAS}

1. Harris M, ClarkJ, Coote N, Fletcher P, et al. British Thoracic Society guidelines for the management of community acquired pneumonia in children: update 2011. Thorax 2011;66 Suppl2:ii1-23.

2. Bradley JS, Byington CL, Shah SS, Alverson B, et al. The management of community-acquired pneumonia in infants and children older than 3 months of age: clinical practice guidelines by the Pediatric Infectious Diseases Society and the Infectious Diseases Society of America. Clin Infect Dis 2011;53(7):e25-76.

3. González Pena H, Ferrero F. El difícil diagnóstico de la simple neumonía. Arch Argent Pediatr 2009;107(6):483-4.

4. Ferrero F, Nascimento-Carvalho CM. Clinical prediction rules and pediatric infectious diseases. Pediatr Infect Dis $J$ 2012;31(6):628-9.

5. Moreno L, Krishnan JA, Duran P, Ferrero F. Development and validation of a clinical prediction rule to distinguish bacterial from viral pneumonia in children. Pediatr Pulmonol 2006;41(4):331-7.

6. Torres FA, Passarelli I, Cutri A, Leonardelli A, et al. Seguridad de una regla de predicción para el manejo inicial de niños con neumonía tratados en forma ambulatoria. Arch Argent Pediatr 2010;108(6):511-5.

7. Torres FA, Pasarelli I, Cutri A, Ossorio MF, et al. Impact assessment of a decision rule for using antibiotics in pneumonia: a randomized trial. Pediatr Pulmonol 2014;49(7):701-6.

8. Laupacis A, Sekar N, Stiell IG. Clinical prediction rules. A review and suggested modifications of methodological standards. JAMA 1997;277(6):488-94.

9. Reilly BM, Evans AT. Translating clinical research into clinical practice: impact of using prediction rules to make decisions. Ann Intern Med 2006;144(3):201-9.

10. Gentile A, Bardach A, Ciapponi A, Garcia-Marti S, et al. Epidemiology of community-acquired pneumonia in children of Latin America and the Caribbean: a systematic review and meta-analysis. Int J Infect Dis 2012;16(1):e5-15.

11. Farrell DJ, Klugman KP, Pichichero M. Increased antimicrobial resistance among nonvaccine serotypes of Streptococcus pneumoniae in the pediatric population after the introduction of 7-valent pneumococcal vaccine in the United States. Pediatr Infect Dis J 2007;26(2):123-8.

12. World Health Organization. Technical bases for the WHO recommendations on management of pneumonia in children at first level health facilities. Geneva, 1991.

13. Puiggari J, Pawluk V. Neumonía aguda. En Voyer LE, ed. Criterios de diagnóstico y tratamiento en pediatría. Buenos Aires: Journal;2006;439-49.

14. Comité Nacional de Neumonología, Subcomisión de Epidemiología, Comité Nacional de Infectología, Comité Nacional de Medicina Interna. Recomendaciones para el diagnóstico y tratamiento de las infecciones respiratorias agudas bajas en menores de 2 años. Arch Argent Pediatr 2006;104(2):159-76.

15. Jain S, Williams DJ, ArnoldSR, AmpofoK, etal. Communityacquired pneumonia requiring hospitalization among U.S. children. N Engl J Med 2015;372(9):835-45.

16. American Academy of Pediatrics. Management of community-acquired pneumonia (CAP) in infants and children older than 3 months of age. Pediatrics 2011;128(6):e1677.

17. Bernztein R, Drake I. Neumonía de la comunidad en niños: 
impacto sanitario y costos del tratamiento en el primernivel de atención público de la Argentina. Arch Argent Pediatr 2009;107(2):101-10.

18. Bernztein R, Drake I, Elordi S. Variabilidad en el manejo de la bronquiolitis en el primer nivel de atención público de la Argentina. Arch Argent Pediatr 2008;106(3):205-11.

19. Kronman MP, Hersh AL, Feng R, Huang YS, et al. Ambulatory visit rates and antibiotic prescribing for children with pneumonia, 1994-2007. Pediatrics 2011;127(3):411-8.

20. Costelloe C, Metcalfe C, Lovering A, Mant D, et al. Effect of antibiotic prescribing in primary care on antimicrobial resistance in individual patients: systematic review and meta-analysis. BMJ 2010;340:c2096.

21. Friedman CR, Whitney CG. It's time for a change in practice: reducing antibiotic use can alter antibiotic resistance. J Infect Dis 2008;197(8):1082-3.

22. Mainous AG 3rd, Diaz VA, Matheson EM, Gregorie $\mathrm{SH}$, et al. Trends in hospitalizations with antibioticresistant infections: U.S., 1997-2006. Public Health Rep 2011;126(3):354-60.

23. Di Fabio JL, Castañeda E, Agudelo CI, De La Hoz F, et al. Evolution of Streptococcus pneumoniae serotypes and penicillin susceptibility in Latin America, Sireva-Vigia Group, 1993 to 1999. PAHO Sireva-Vigia Study Group. Pan American Health Organization. Pediatr Infect Dis J 2001;20(10):959-67.

24. Cardoso MR, Nascimento-Carvalho CM, Ferrero F, Berezin EN, et al. Penicillin-resistant pneumococcus and risk of treatment failure in pneumonia. Arch Dis Child 2008;93(3):221-5.

25. Shah S, Bachur R, Kim D, Neuman MI. Lack of predictive value of tachypnea in the diagnosis of pneumonia in children. Pediatr Infect Dis J 2010;29(5):406-9.
26. Courtoy I, Lande AE, Turner RB. Accuracy of radiographic differentiation of bacterial from nonbacterial pneumonia. Clin Pediatr (Phila) 1989;28(6):261-4.

27. Swingler GH. Radiologic differentiation between bacterial and viral lower respiratory infection in children: a systematic literature review. Clin Pediatr (Phila) 2000;39(11):627-33.

28. Khamapirad T, Glezen WP. Clinical and radiographic assessment of acute lower respiratory tract disease in infants and children. Semin Respir Infect 1987;2(2):130-44.

29. Torres F, Chiolo MJ, González N, Durán P, et al. Capacidad para predecir etiología con la radiografía de tórax en niños hospitalizados con neumonía. Arch Arg Pediatr 2006;104(2):106-8

30. Swingler GH. Observer variation in chest radiography of acute lower respiratory infections in children: a systematic review. BMC Med Imaging 2001;1(1):1.

31. FerreroF,TorresF,NoguerolE, GonzálezN, etal.Evaluación de dos métodos estandarizados de interpretación de radiografías de tórax en niños con neumonía. Arch Argent Pediatr 2008;106(6):510-4.

32. López M, Torres F, Davenport C, Rial MJ, et al. Validación de una regla de predicción simplificada para la presunción de etiología en niños con neumonía. Arch Argent Pediatr 2011;109(6):499-503.

33. World Health Organization. Standardization of Interpretation of chest radiographs for the diagnosis of pneumonia in children. Geneva, 2001.

34. Lynch T, Bialy L, KellnerJD, Osmond MH, etal. Asystematic review on the diagnosis of pediatric bacterial pneumonia: when gold is bronze. PLoS One 2010;5(8):e11989.

35. Maguire JL, Kulik DM, Laupacis A, Kuppermann N, et al. Clinical prediction rules for children: a systematic review. Pediatrics 2011;128(3):e666-77.

El progreso de la medicina nos depara

el fin de aquella época liberal en la que

el hombre aún podía morirse de lo que quería.

Stanislaw Lec

(1909-1966) 